Case Report:

\title{
Reversal of Degenerative Changes in Cervical Spine Following Bipolar Release in a Neglected Case of Congenital Muscular Torticollis: A Case Report
}

\author{
Nima Derakhshan ${ }^{1}$ (D) Arefe Rahimikhorrami ${ }^{2^{*}}$ (D)
}

1. MD, Assistant Professor of Neurosurgery, Department of Neurosurgery, Namazi Hospital, Shiraz University of Medical Sciences, Shiraz, Iran 2. MD, Student Research Committee, Department of Neurosurgery, School of Medicine, Shiraz University of Medical Sciences, Shiraz, Iran

$\begin{array}{ll}\begin{array}{c}\text { Use yourdevice to scan } \\ \text { and read the article online }\end{array} & \begin{array}{l}\text { Cftation: Derakhshan N, Rahimikhorrami A. Reversal of Degenerative Changes in Cervical Spine Following Bipolar Re- } \\ \text { lease in a Neglected Case of Congenital Muscular Torticollis: A Case Report. Iran J Neurosurg. 2020; 6(2):89-92. http://dx.doi. } \\ \text { org/10.32598/irjns.6.2.6 }\end{array} \\ \text { dol: }: \text { http://dx.doi.org/10.32598/irjns.6.2.6 }\end{array}$

\section{(i) (\$)}

Article info:

Received: 10 Jan 2020

Accepted: 12 Mar 2020

Available Online: 01 Apr 2020

Keywords:

Spasmodic torticollis, Disc degeneration, Spine, Cervical vertebrae

\section{ABSTRACT}

Background \& Importance: Congenital Muscular Torticollis (CMT) is a common cause of torticollis in children. Despite the easy diagnosis, rare cases may be neglected and untreated during the management of the patients, which can not only impose cosmetic problems for them but also affect the cervical spine with accelerated degeneration. Most patients with CMT can be managed non-surgically with medical and physical therapies but surgery is indicated in some cases when non-surgical attempts are unsuccessful.

Case Presentation: Herein, we are reporting a 16-year-old female with neglected CMT, and neck pain secondary to severe degenerative changes. We believe that neglected and untreated CMT cases may present with accelerated spine degeneration and surgical intervention should be considered promptly to reverse this process

Conclusion: Surgical intervention not only produces good cosmetic results but also reverses the degeneration process and protects the patient from disabling deformities later in life.

\section{* Corresponding Author:}

Arefe Rahimikhorrami, MD.

Address: Student Research Committee, Department of Neurosurgery, School of Medicine, Shiraz University of Medical Sciences, Shiraz, Iran

Tel: +98 (935) 5695377

E-mail:dr.rahimikhorrami@gmail.com 


\section{Highlights}

- Neglected Torticollis in adults mandates early surgical intervention.

- Bipolar release of SCM (shortening of the sternocleidomastoid) should be considered in adult cases of CMT (congenital muscular torticollis).

- Surgical release of CMT even in adults has the potential of reversing the progression of degenerative disc disease.

\section{Plain Language Summary}

Congenital muscular torticollis is a condition that presents as turning of the head to one side due to the shortening of SCM, a key muscle involved in neck rotation. If diagnosed early in childhood, there is a higher likelihood of successful treatment with non-surgical measures. However, surgery might be necessary to correct the cervical spine posture as well as facial symmetry. Adult patients who had refused treatment or were neglected to be diagnosed in a timely fashion have to undergo surgical lengthening of the shortened muscle. This report is in favor of the role of surgical release to increase the chance of improving cosmetic results as well as the reversal of early stages of degeneration in the spine.

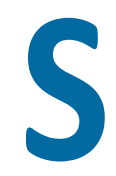

\section{Introduction}

pasmodic or Congenital Muscular Torticollis (CMT) is a postural deformity, typically characterized by the cock-robin postural deformity which is lateral flexion of the neck to the same side and rotation of the neck to the opposite side. This common congenital musculoskeletal anomaly occurs due to the unilateral shortening of the Sternocleidomastoid (SCM) muscle [1]. If diagnosed in a timely fashion, CMT can be managed with full recovery with medical and physical therapies without the necessity for surgery in most cases [2]. Surgical intervention is reserved for patients who fail to respond to non-surgical treatments and those who suffer recurrences following previous treatment [3]. CMT can rarely be seen in adults if the patients defer treatment and in situations that patients are neglected due to economic, cultural, and social shortcomings [4].

\section{Case Presentation}

A 16-year-old female who was a known case of CMT since birth was visited in the neurosurgery clinic. Even though her family was aware of her condition, they did not follow treatment protocols due to their low socioeconomic status. However, after beginning high school, she began to worry about her cosmetic appearance and her neck pain got worse with a Visual Analog Scale (VAS) of 8 from 10.
Although the fibrotic band over the left SCM and the cock-robin position of the head confirmed the diagnosis of CMT, a cervical spine x-ray was requested due to her severe neck pain. The lateral neck $\mathrm{X}$-ray revealed the loss of lordosis and kyphotic angulation of $9{ }^{\circ}$ at the C4-C5 level, mild C4-C5 anterolisthesis, a $30^{\circ}$ scoliosis measured by the Cobb angle between $\mathrm{C} 3$ and $\mathrm{C} 6$ and the apex at C4-C5, as well as C4 and C5 osteophyte formation. The radiograph did not show any mal-developments in the cervical vertebrae (Figure $1 A, B, \& C$ ). Clinical and radiologic examination of the thoracolumbar spine was unremarkable. On physical examination, she had almost zero Range of Motion (ROM) of contralateral neck rotation. The neurologic exam was intact and the ENT examination was unremarkable as well.

The patient's birth history revealed that she was born via Normal Vaginal Delivery (NVD) from her nullipara 18-year-old mother with vertex presentation, and an unremarkable pregnancy and delivery. The parents had no consanguineous relationship. Her past medical and family history was negative for any other congenital problems.

\section{Surgical intervention}

Due to the severity of torticollis, the bipolar release of the SCM (without Z-plasty lengthening) was carried out. A 1-cm transverse incision was made 2 fingerbreadths behind the ear just below the mastoid tip. Another 4-cm incision parallel to cervical skin creases was made one fingerbreadth proximal to the sternoclavicular joint. During the operation, all of the sclerotic muscle fibers 


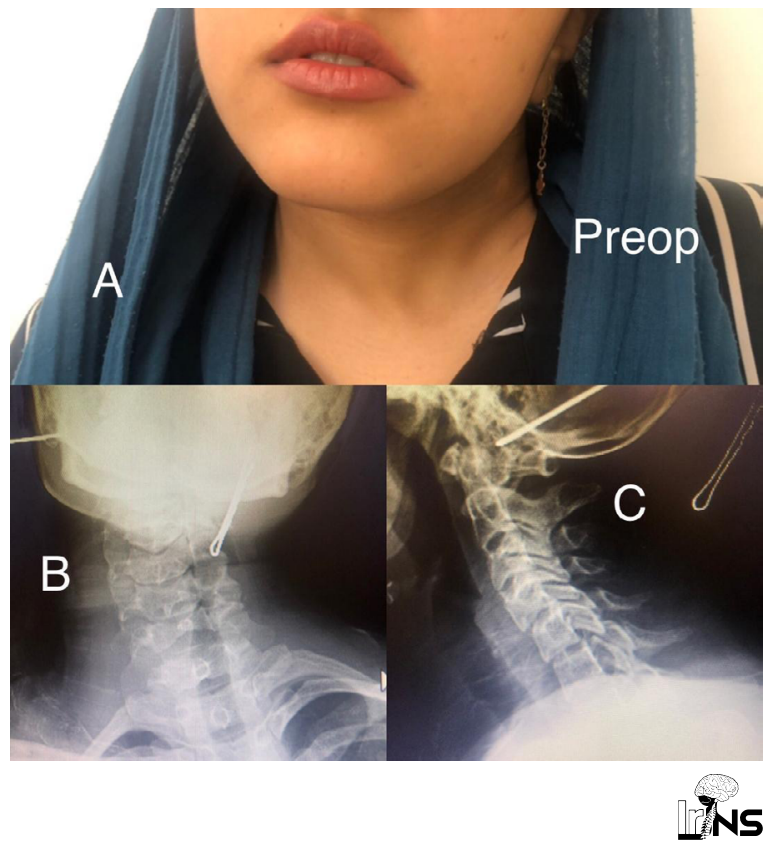

Figure 1. A pre-operative image of a 16-year-old female with muscular torticollis

A: Showing the well-known cock robin position. Anterior-Posterior (AP); B: and lateral C: Cervical X-ray revealing the loss of lordosis and kyphotic angulation of $9^{\circ}$ at the C4-C5 level, mild C4-C5 anterolisthesis, $30^{\circ}$ of coronal plane deformity with its vertex on C4$\mathrm{C} 5$ as well as osteophyte formation on $\mathrm{C} 4$ and $\mathrm{C} 5$ bodies.

were released from proximal and distal insertion points and after confirming the full ROM of rotation and lateral flexion on either side, the surgical sites were closed in anatomical layers.

\section{Post-operative results and follow-up}

Following the operation, she was advised to use a hard collar for 2 weeks and begin the physical therapy thereafter with neck exercises including contralateral manual stretching. At the 2-month follow-up visit, the patient had VAS of 0 from 10, the kyphotic angulation at C4-C5 was reduced to $0{ }^{\circ}$ and her AP X-ray showed normal alignment in the coronal plane (Figure $2 A, B, \& C$ ). Despite the minimal limitation of ROM on active rotation and lateral flexion, full ROM could be obtained with gentle force on passive motion. The patient had excellent cosmetic results and only a minimal scar left on each incision site.

\section{Discussion}

Surgical treatment of neglected CMT patients can be difficult due to adjacent tissue contractures, adhesions, and extensive fibrosis $[5,6]$. Best outcomes are obtained between 1 to 4 years of age group. After 5 years of age, the efficacy of

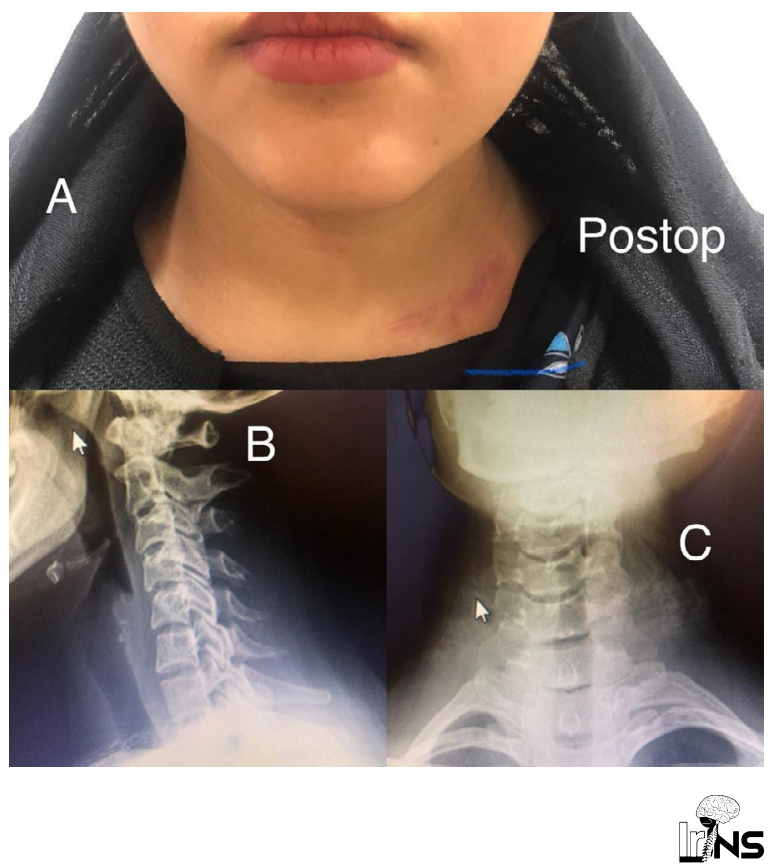

Figure 2. AP X-ray results

A: Two months after operation showing the corrected posture; $B$ : Lateral and AP; C: Cervical X-rays after two months showing normal alignment in coronal plane and reversal of degenerative changes.

surgery is reduced due to irreversible craniofacial deformity [7-9].

Neglected cases of CMT require surgery for the release of tethered elements and conservative treatments are no longer a solution for these patients [3]. Surgical techniques for SCM release are unipolar release, bipolar release, and bipolar release with Z-plasty lengthening [4]. Age, the severity of symptoms, and the surgeon's preference are the factors to be considered for choosing the technique when surgical intervention is indicated [4].

Younger patients and those with moderate severity may be managed by the unipolar release which provides the least invasion and best cosmetic results. However, this technique is not suitable for patients with severe deformity due to the higher possibility of recurrence [4].

Bipolar release with or without Z-plasty lengthening should be considered as the preferred technique for patients with severe deformity and neglected cases whose treatment is deferred until adulthood $[3,10]$.

Herein, we report our experience with the surgical treatment of a neglected case of CMT. Our findings support the idea that surgical release should be performed promptly upon the diagnosis for neglected CMT patients. Surgical in- 
tervention is beneficial in terms of pain relief as well as the reversal of degenerative changes in the cervical spine.

\section{Conclusion}

Neglected and untreated CMT cases may present with accelerated spine degeneration and surgical intervention should be considered promptly to reverse this process. Surgical intervention not only leads to good cosmetic results but also reverses the degeneration process and protects the patient from disabling deformities later in life. Although surgery might not ca optimal results in terms of craniofacial asymmetry and gaze problems in neglected and adult cases, compared with those operated at a younger age, it remains the only viable option to treat neck pain and correct the deformity.

\section{Ethical Considerations}

Compliance with ethical guidelines

Written informed consent was obtained from the patient for publication of this case report and accompanying images.

\section{Funding}

This research did not receive any grant from funding agencies in the public, commercial, or non-profit sectors.

\section{Authors' contributions}

Both authors contributed equally in all stages of the study.

Conflict of interest

The authors declared no conflict of interest.

\section{References}

[1] Petronic I, Brdar R, Cirovic D, Nikolic D, Lukac M, Janic D, et al. Congenital muscular torticollis in children: Distribution, treatment duration and outcome. European Journal of Physical and Rehabilitation Medicine. 2009; 46(2):153-7. [PMID]

[2] Moussaoui A, EnnouhiMA, Guerrouani A. A rare case of neglected congenital muscular torticollis in an adult and review of literature. British Journal of Medicine and Medical Research. 2015; 7(6):541-9. [DOI:10.9734/BJMMR/2015/15439]

[3] Omidi-Kashani F, Hasankhani EG, Sharifi R, Mazlumi M. Is surgery recommended in adults with neglected congenital muscular torticollis? A prospective study. BMC Musculoskeletal Disorders 2008; 9(1):158 [DOI:10.1186/1471-2474-9-158] [PMID] [PMCID]
[4] Lepetsos P, Anastasopoulos PP, Leonidou A, Kenanidis E Flieger I, Tsiridis E, et al. Surgical management of congenital torticollis in children older than 7 years with an average 10-year follow-up. Journal of Pediatric Orthopaedics B. 2017; 26(6):580-4. [DOI:10.1097/BPB.0000000000000323] [PMID]

[5] Uçar BY. The efficacy of surgical treatment for neglected congenital muscular torticollis. EC Orthopaedics. 2019; 10(2): 75-8. https://www.researchgate.net/profile/Bekir_Ucar/ publication/331212050

[6] Wang JL, Qi W, Liu YJ. Endoscopic release of congenital muscular torticollis with radiofrequency in teenagers. Journal of Orthopaedic Surgery and Research. 2018; 13(1):100. [DOI:10.1186/s13018-018-0801-6] [PMID] [PMCID]

[7] Lim KS, Shim JS, Lee YS. Is sternocleidomastoid muscle release effective in adults with neglected congenital muscular torticollis? Clinical Orthopaedics. 2014; 472(4):1271-8. [DOI:10.1007/s11999-013-3388-6] [PMID] [PMCID]

[8] Min KJ, Ahn AR, Park EJ, Yim SY. Effectiveness of surgical release in patients with neglected congenital muscular torticollis according to age at the time of surgery. Annals of Physical and Rehabilitation Medicine. 2016; 40(1):34-42. [DOI:10.5535/arm.2016.40.1.34] [PMID] [PMCID]

[9] Lee GS, Lee MK, Kim WJ, Kim HS, Kim JH, Kim YS. Adult patients with. Congenital muscular torticollis treated with bipolar release: Report of 31 cases. Journal of Korean Neurosurgical Society 2017; 60(1):82. [DOI:10.3340/jkns.2016.0303.018] [PMID] [PMCID]

[10] Patwardhan S, Shyam AK, Sancheti P, Arora P, Nagda T, Naik P. Adult presentation of congenital muscular torticollis:a series of 12 patients treated with a bipolar release of sternocleidomastoid and Z-lengthening. Journal of Bone and Joint Surgery B. 2011; 93(6):828-32. [DOI:10.1302/0301620X.93B6.26232] [PMID] 